\title{
Cancer genetics and risk assessment: the practicing clinician's role
}

\begin{abstract}
Genetic counseling and testing is now part of our day to day oncology practice and has the potential to reduce incidence and mortality of cancer in high-risk individuals. This can be conducted by properly trained physicians and other practitioners to maximize identification of high risk individuals and reduce unnecessary or inappropriate testing.
\end{abstract}

Keywords: cancer prevention, cancer risk assessment, clinical genetics, genetic counseling
Volume I Issue I - 2014

\section{Carlos A Encarnacion}

Texas Oncology, USA

Correspondence: Carlos A Encarnacion, Texas Oncology, 1700 W Highway 6, Waco, Texas 767I2, USA, Tel 254-399-074I, Fax 254-399-5939, Email cencarnacion@stcglobal.net

Received: May 15, 2014 | Published: May 24, 2014
As Gregor Mendel worked on a theory to explain his observations, he could not have foreseen its impact on our current approach to cancer risk assessment and prevention. The last several years gave us a deeper understanding of the role played by genes such as BRCA (and others) in cancer development. This growing list of high risk genes created an expanding population of individuals whose cancer risk is elevated but modifiable. Because the fields of genetics and oncology are inextricably ingrained, all health professionals involved in cancer care need a solid understanding of these concepts. The application of such knowledge by properly trained individuals of different disciplines, especially practicing clinicians, should expand access to genetic counseling for people at risk.

Most of us realize the importance of genetics in the evaluation and modification of cancer risk. How can we not? Identification of carriers can lead to prevention or early detection of malignancies and result in reduced mortality for these individuals, not to mention a better use of health care resources. These mutations are not as rare as we may think: the prevalence of deleterious BRCA 1 or 2 mutations in the general population of European ancestry is about $0.25 \%{ }^{1-3}$ That is close to a million people in the U.S. and does not take in consideration the higher rates seen in certain ethnic groups or the many other genes known to increase the risk of different cancers. However, in order to be effective in cost and results, genetic risk assessment needs to be conducted by professionals with solid knowledge of the current guidelines for testing and risk management as well as good counseling skills. Lack of this, together with external pressure from patients, media, and clinical laboratories can result in a dreadful, costly mess.

In an early educational meeting at the 2010 San Antonio Breast Cancer Symposium, Dr. Sharon Plon and colleagues presented the results of their physician survey evaluating the way different practitioners (family medicine, general surgeons, OB/GYNs, oncologists, etc.) prescribe genetic testing. It showed that even among specialists there is a lack of understanding of the basic concepts of genetic testing and its application to clinical practice. ${ }^{4}$ Through the years, I have witnessed colleagues engage in unnecessary and disorganized testing of patients while missing true high risk individuals. It is not surprising then, to hear some in the genetics field suggest that medical practitioners (physicians, nurse practitioners, etc.) should stay away from genetic counseling. I understand but respectfully disagree. Practicing clinicians have a huge opportunity to identify and interact with a large number of individuals at risk.
Furthermore, because certified counselors can be a rare commodity in certain areas, patients sometimes have delayed or limited access to counseling.

Rather than exclusion, the answer is cooperation and inclusion. Groups such as US Oncology's Genetic Risk Evaluation and Testing (GREAT) Program and the Genetics Program at City of Hope give physicians and other medical practitioners the knowledge and skills to manage the most common risk assessment and management situations. Patients with very complex cases can be discussed in interdisciplinary boards or referred to higher level genetic counseling if necessary. These programs have shown that improving the rate of identification of people at risk by clinicians in a community setting is feasible and practical..$^{5}$ Many of such programs exist through the U.S. and in my opinion offer an expanded and systematic access to genetic counseling to patients. Genetics and oncology are now lifelong partners and it is our obligation to improve our ability to manage the expanding population of high risk individuals.

\section{Conclusion}

It is very exciting to contemplate on the potential benefits stemming from our ever growing knowledge in the field of genetics. Practicing clinicians can play a pivotal role in genetic counseling but in a way that benefits the greatest number of individuals while at the same time being good stewards of our available health care resources.

\section{Acknowledgments}

None.

\section{Conflicts of interest}

Author declares there are no conflicts of interest.

\section{References}

1. Ford D, Easton DF, Bishop DT, et al. Risk of cancer in BRCA mutation carriers. Lancet. 1994;343(8899):692-695.

2. Claus EB, Schildkraut JM, Thompson WD, et al. The genetic attributable risk of breast and ovarian cancer. Cancer. 1996;77(11):2318-2324.

3. Whittemore AS, Gong G, Itnyre I. Prevalence and contribution of BRCA1 mutations in breast cancer and ovarian cancer: results from three U.S. population-based case-control studies of ovarian cancer. Am J Hum Genet. 1997;60(3):496-504. 
4. Dhar SU, Cooper HP, Wang T, et al. Significant differences among physician specialties in management recommendations of BRCA 1 mutation carriers. Breast Cancer Res Treat. 2011;129(1):221-227.
5. Langer L, Clark L, Gress J, et al. A structured genetic risk evaluation and testing program in the community oncology practice increases identification of individuals at risk for BRCA mutations. Cancer Research. 2012;72(24 Suppl). 